\title{
Effect of Reactive Amphiphiles on the Silicate Dispersion and Degradation Behavior of ABS/layered Silicate Nanocomposites
}

\author{
By Jong Hyun KIM, ${ }^{1}$ Kwanghyon KIM, ${ }^{1}$ Youn Cheol KIM, ${ }^{2}$ and In Jae CHUNG ${ }^{2, *}$
}

Acrylonitrile-butadiene-styrene copolymer (ABS)/layered silicate nanocomposites were synthesized by in situ emulsion polymerization and the effect of reactive amphiphiles on the silicate dispersion was investigated. Three reactive amphiphiles with different hydrophobic chain lengths were synthesized (DC2, DC6, and DC12). ABS/layered silicate nanocomposites showed intercalated states of silicates when the reactive amphiphiles with short hydrophobic chain (DC2 or DC6) were added. On the other hand, the silicates were exfoliated in ABS matrix for the nanocomposite with the long chain amphiphiles (DC12). And we verified that silicate layers were attached on the surface of the graft ABS particles by using SEM and TEM. The nanocomposite obtained by using DC12 produced a slightly larger amount of residue than those by DC2 and DC6 and its decomposition temperature was also higher. Therefore it could be concluded that reactive amphiphiles played an important role in the dispersibility of silicate and the degradation behavior of ABS/layered silicate nanocomposite.

KEY WORDS: Layered Silicate / Acrylonitrile-butadiene-styrene (ABS) Terpolymer / Reactive Amphiphiles /

Polymer/layered silicate nanocomposites (PLSNs) are a class of hybrid materials composed of inorganic layered silicates with nanometer-scale dimensions imbedded in an organic polymer matrix. ${ }^{1-6}$ They have attracted technological and scientific interest in recent years, because they have shown the dramatic increase in physical, thermal, and mechanical properties even though the loading of silicate is low. ${ }^{7-9}$ The physical properties are presumably enhanced due to the synergistic effects of the enormous increase in surface of silicate particles and the maximized interaction between the fillers and polymer molecules.

There are various methods to delaminate the silicate layers and to get PLSNs with a good dispersion of silicate in polymer matrix, such as melt intercalation, ${ }^{10-13}$ in situ polymerization, ${ }^{14-18}$ and curing systems. ${ }^{19-21}$ The layers of natural silicates have strong interaction between them due to negative charges and hydrogen bond in their crystal structures. ${ }^{22,23}$ The basal space of pristine silicate is about $1 \mathrm{~nm}$, which is smaller than the radius of gyration of general polymers. This might be an obstacle for polymers to penetrate into or delaminate silicate layers. So, most hydrophobic polymers have limitation for their penetration into hydrophilic silicate layers. Up to now, the preparation of PLSNs has been mainly based on organically modified layered silicates ${ }^{24-28}$ with various alkylammoniums, showing hydrophobic characters.

Acrylonitrile-butadiene-styrene copolymer (ABS) is a wellknown commercial thermoplastic polymer for excellent mechanical properties. ${ }^{29,30}$ In spite of the industrial significance, ABS/layered silicate nanocomposite has not been intensively researched except few articles. ${ }^{31-33}$ Lee et al. ${ }^{31}$ reported that intercalated ABS/sodium montmorillonite nanocomposites were synthesized with emulsion polymerization. Wang et al. ${ }^{32}$ prepared intercalated or exfoliated structure of nano-ABS with an organically modified clay through melt blending method. And our group has tried to obtain the exfoliated ABS/layered silicate nanocomposites through an in situ polymerization using a reactive amphiphiles, 2-acrylamido-2-methyl-1-propanesulfonic acid (AMPS), which has amido and vinyl groups to form strong interaction with pristine silicates and make the polymer end-tethered on the silicate layers. ${ }^{33}$ And we pointed out that silicate works as a colloidal stabilizer due to several factors during ABS emulsion polymerization.

In this article, we want to prepare exfoliated ABS/layered silicate nanocomposites and elucidate the role of reactive amphiphile on the dispersibility of silicate in ABS matrix through in situ polymerization with newly synthesized reactive amphiphiles which had different hydrophobic chain lengths.

\section{EXPERIMENTAL}

\section{Materials}

The layered silicate is Cloisite-Na from Southern Clay Products with a cation exchange capacity (CEC) of $92.6 \mathrm{meq} /$ $100 \mathrm{~g}$. Its primary particle size is about $150 \mathrm{~nm}$. Pristine silicate was dispersed in deionized water for $24 \mathrm{~h}$ at ambient temperature before polymerization. Acrylonitrile (AN), styrene (S), cumene hydroperoxide as an initiator, and Dodecylbenzenesulfonic acid sodium salt (DBS-Na) were purchased from Aldrich and used without further purification. Polybutadiene (PBD) latex (52 wt \% in water, average particle size is $250 \mathrm{~nm}$ ) was supplied from Samsung Cheil Industries. For the synthesis of reactive amphiphiles used in this article, $N, N$-dimethylaminoethyl methacrylate (DMAEMA) and 1-bromoalkanes were purchased from Aldrich.

\footnotetext{
${ }^{1}$ Department of Chemical and Biomolecular Engineering, Korea Advanced Institute of Science and Technology, 373-1, Guseong-dong, Yuseong-gu, Daejeon, Republic of Korea

${ }^{2}$ Major in Polymer Engineering, Kongju National University, 275, Budae-dong, Cheonan, Chungnam 330-717, Korea

*To whom correspondence should be addressed (E-mail: chung@kaist.ac.kr).
} 


\section{Synthesis of Reactive Amphiphiles}

Reactive amphiphiles were synthesized by direct reaction of DMAEMA with the 1-bromoethane, 1-bromohexane, and 1-bromododecane. Typically DMAEMA was mixed with 1-bromoalkanes without any additional solvent. After the addition of a small portion of hydroquinone to inhibit any spurious radical polymerization, the mixture was stirred overnight at $50{ }^{\circ} \mathrm{C}$. The solid product was washed several times with dry ether to remove the excess DMAEMA. The products were named DC2, DC6, and DC12 according to the starting materials, 1-bromoethane, 1-bromopentane, and 1-bromoundecane, respectively.

\section{Polymerization of ABS/layered Silicate Nanocomposites} Polymerization was Carried Out in Two Stages. 1st stage. $200 \mathrm{~g}$ of water, $1.5 \mathrm{~g}$ of reactive amphiphiles, $25 \mathrm{~g}$ of DBS-Na aqueous solution (DBS-Na/water $=2.5 \mathrm{~g} / 22.5 \mathrm{~g}$ ), aqueous silicate dispersion (silicate/water $=5 \mathrm{~g} / 200 \mathrm{~g}$ ), and $76.4 \mathrm{~g}$ of PBD latex emulsion were stirred at room temperature in a $1 \mathrm{~L}$ four-neck round bottom flask. The flask was equipped with a baffle stirrer, a reflux condenser, a nitrogen inlet, and a rubber septum. $0.2 \mathrm{~g}$ of cumene hydroperoxide dissolved in $15 \mathrm{~g}$ of $\mathrm{AN}$ monomer was introduced into the reactor. The mixture was stirred at $200 \mathrm{rpm}$ for $30 \mathrm{~min}$ under nitrogen atmosphere at room temperature. Then the reactor was heated to $60^{\circ} \mathrm{C}$ and stirred for $30 \mathrm{~min}$ to make PBD swollen with AN monomer. And then initial polymerization was performed at $70^{\circ} \mathrm{C}$ for $15 \mathrm{~min}$.

2nd stage. After the initial polymerization was completed, $45 \mathrm{~g}$ of mixture of monomers $(\mathrm{S} / \mathrm{AN}=30 \mathrm{~g} / 15 \mathrm{~g}$ ) including $0.2 \mathrm{~g}$ of cumene hydroperoxide was fed at the rate of $0.24 \mathrm{cc} /$ min into the flask through the rubber septum by using a syringe pump. After monomer feeding was finished, the reaction temperature was increased to $75^{\circ} \mathrm{C}$, and maintained for additional $1 \mathrm{~h}$ to complete the reaction. The latex was recovered from the flask and coagulated with $3 \mathrm{wt} \%$ of $\mathrm{MgSO}_{4}$ aqueous solution at $60^{\circ} \mathrm{C}$, and then freeze-dried for $2 \mathrm{~d}$ and further dried in a high vacuum oven at $50{ }^{\circ} \mathrm{C}$ for $3 \mathrm{~d}$.

\section{Sample Code}

Each sample is named like this $\mathrm{C \# -NO}$ or $\mathrm{C \# -X}$. The letter " $\mathrm{C}$ " denotes Cloisite-Na and the number following " $\mathrm{C}$ " means the loading content of Cloisite-Na represented phm (part per hundred monomer). The "NO" means that there is no reactive amphiphiles. And the " $X$ " represents the type of reactive amphiphiles such as DC2, DC6, and DC12. The silicate and reactive amphiphile contents in each sample were summarized in Table I.

\section{Characterization}

To characterize the reactive amphiphiles, ${ }^{1} \mathrm{H}$ nuclear magnetic resonance (NMR) spectra were collected for DC2, DC6, and DC12 using a Bruker AMX $500 \mathrm{MHz}$ spectrometer employing $\mathrm{CDCl}_{3}$ as the solvent. X-Ray diffraction (XRD) and transmission electron microscope (TEM) were used to examine the dispersion state of silicates in composites. XRD measure-
Table I. Formulations and sample codes of the synthesized materials

\begin{tabular}{cccc}
\hline Sample code & $\begin{array}{c}\text { Cloisite-Na } \\
\left(\mathrm{phm}^{*}\right)\end{array}$ & $\begin{array}{c}\text { Reactive Amphiphiles } \\
\text { (phm) }\end{array}$ & $\begin{array}{c}\text { DBS-Na } \\
\text { (phm) }\end{array}$ \\
\hline C0-NO & 0 & 0 & 2.5 \\
C0-DC12 & 0 & $1.5(\mathrm{DC12})$ & 2.5 \\
C5-NO & 5 & 0 & 2.5 \\
C5-DC2 & 5 & $1.5(\mathrm{DC} 2)$ & 2.5 \\
C5-DC6 & 5 & $1.5(\mathrm{DC6})$ & 2.5 \\
C3-DC12 & 3 & $1.5(\mathrm{DC12})$ & 2.5 \\
C5-DC12 & 5 & $1.5(\mathrm{DC} 12)$ & 2.5
\end{tabular}

*phm: parts per hundred monomer.

ment was carried out by using a Rigaku X-ray generator $(\mathrm{CuK} \alpha$ radiation with $\lambda=0.15406 \mathrm{~nm}, 40 \mathrm{kV}$ and $80 \mathrm{~mA})$ at room temperature. The diffractograms were obtained at the scattering angles from $1.2^{\circ}$ to $10^{\circ}$ at a scanning speed of $2^{\circ} / \mathrm{min}$. Transmission electron microscope and EDAX, Pillips CM20, was used at an acceleration voltage of $160 \mathrm{kV}$. An ultra thin section of about $70 \mathrm{~nm}$ in thickness was prepared by an RMC ultramicrotome at room temperature. Images of the prepared latex particles were obtained using a Sirion scanning electron microscope (SEM). Samples for SEM analysis were prepared by dispersing a few drops of latex into triple-distilled water. This dilute latex was sonicated for $30 \mathrm{~min}$ in order to obtain homogeneous dispersions, and then one drop of latex was placed on a silicon wafer and evaporated slowly. And then the sample was coated with Pt film of $10 \mathrm{~nm}$ thickness under ultra-high vacuum. Thermogravimetric analysis (TGA) was performed with a Perkin-Elmer thermobalance by heating from $30^{\circ} \mathrm{C}$ to $600^{\circ} \mathrm{C}$ with the rate of $10^{\circ} \mathrm{C} / \mathrm{min}$ under $\mathrm{N}_{2}$ atmosphere.

\section{RESULTS AND DISCUSSION}

\section{Characterization of Reactive Amphiphiles}

Figure 1 represents chemical structure and ${ }^{1} \mathrm{H}$ NMR spectrum of DC12. Each proton shows the proper chemical shifts $(\delta)$ and its integration value exactly corresponds to the number of protons. DC2 and DC6 show similar results with DC12 in chemical shift and integration value.

\section{Modification of Silicate with Reactive Amphiphiles}

To check the cation-exchange capability of the synthesized reactive amphiphiles, Cloisite-Na was modified with DC2, DC6, and DC12. Cloisite-Na (1 g) was dispersed into $100 \mathrm{~g}$ of water for $24 \mathrm{~h}$. DC2 $(0.295 \mathrm{~g})$, DC6 $(0.358 \mathrm{~g})$ and DC12 $(0.452 \mathrm{~g})$ were dissolved into $100 \mathrm{~g}$ of water, respectively. And then each reactive amphiphile aqueous solution was poured into the aqueous silicate dispersion under vigorous stirring for 10 min by using magnetic stirrer. A white precipitate was formed. The precipitate was collected on a filter paper, washed with water several times, and freeze-dried to produce a modified Cloisite-Na with reactive amphiphiles (C/DC2, C/DC6, and C/DC12). As a result, the silicates with a hydrophobically modified surface were obtained. Reactive amphiphiles can facilitate the intercalation of a hydrophobic 

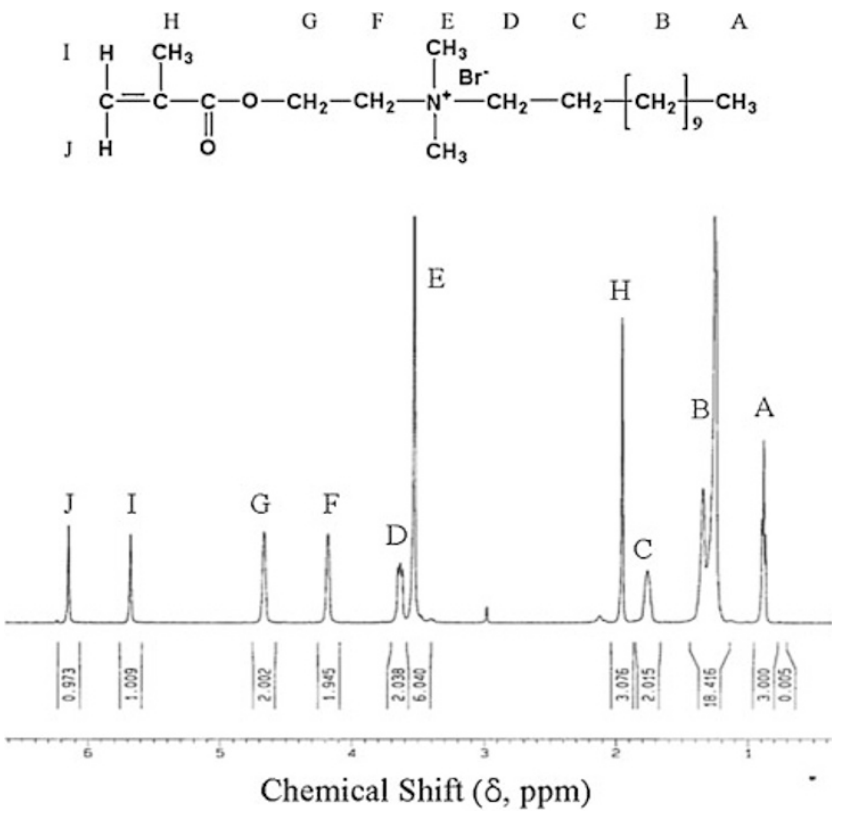

Figure 1. Chemical structure and ${ }^{1} \mathrm{H}$ NMR spectrum of DC12.

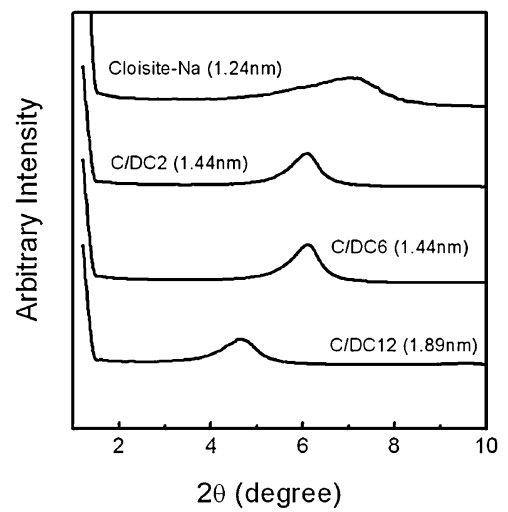

Figure 2. XRD patterns of pristine Cloisite-Na and modified Cloisite-Na with DC2, DC6, and DC12.

monomer into silicate by reducing the surface energy and make the modified Closite-Na formed between silicate sheets. XRD was used to show how the silicates were intercalated by reactive amphiphiles with different chain lengths. Figure 2 shows the XRD patterns of pristine and modified silicates with reactive amphiphiles. The $d$-spacing was obtained from the peak position $\left(d_{001}-\right.$ reflection) of WAXD traces by using Bragg's equation:

$$
2 d_{001} \sin \theta=\lambda
$$

where $d_{001}$ is the interplanar distance of (001) reflection plane, $\theta$ is the diffraction angle, and $\lambda$ is the wavelength. The interlayer distance of pristine silicate is $1.24 \mathrm{~nm}$ in dry state. And modified silicates with DC6 and DC12 had those of $1.44 \mathrm{~nm}$ and $1.89 \mathrm{~nm}$, respectively. This suggests that the reactive amphiphiles can successfully modify silicate and DC12 makes the gallery wider because it has longer hydrophobic tail than DC2 and DC6.

\section{Emulsion Morpholoy}

The silicate layers can not penetrate into the PBD latex during the synthesis of ABS/layered silicate nanocomposites, because PBD latex is a cross-linked hydrophobic rubber but natural silicate is hydrophilic with a size more than one hundred $\mathrm{nm}$. So we think that if the silicates are modified with reactive amphiphiles, the silicate layers may be adsorbed on the PBD latex surface owing to the hydrophobic character of organically treated silicates. To confirm this, SEM was utilized to see the surface morphology of latex particles. Figure 3 shows SEM images for the particles obtained by the in situ emulsion polymerization of AN and St in the presence of PBD latex and layered silicate. In the case of (a) g-ABS (CO-NO), there are many round particles with different size and indicating the coexistence of $\mathrm{g}$-ABS and free SAN. The particles over $200 \mathrm{~nm}$ are $\mathrm{g}$-ABS and the others under this size are free SAN. The morphologies of $\mathrm{C} 0-\mathrm{DC} 12$ and $\mathrm{C} 5-\mathrm{NO}$ particles show similar pattern with g-ABS particle. This indicates that each addition of reactive amphiphile or silicate does not affect the morphology change during the polymerization. Especially in the image of (c) C5-NO, we can not find any disc-like structures considered as silicate layers. But in the case of (d) C5-DC12, the particles have rough surfaces and the silicates may be adhered on the surfaces of the polymer particle. This fact tells that reactive amphiphile helps silicates adhere onto the surface of the g-ABS particle.

\section{Silicate Morphology}

The dispersion state of silicate layers in the polymer matrix is usually examined by XRD and TEM. In order to see the effect of reactive amphiphile on the dispersibility of silicate in the ABS polymer matrix, we prepared ABS/layered silicate nanocomposites with different reactive amphiphiles and their XRD profiles are shown in Figure 4. In the case of samples prepared by using short reactive amphiphiles (DC2 and DC6), the (001) plane peak of silicate can be seen at around $2 \theta=6.10^{\circ}$ and its basal spacing is $1.44 \mathrm{~nm}$, which is $0.20 \mathrm{~nm}$ increment when compared with pristine Cloisite-Na. However the peak for C5-DC12 was not seen, which indicate the exfoliation of silicate layers in the ABS matrix. From these results, it is found that the reactive amphiphile with a longer hydrophobic tail has a better influence on the exfoliation of silicate layers than that with a shorter one.

Figure 5 shows TEM images of ABS/layered silicate nanocomposite prepared by melt-blending C5-DC12 and SAN using a miniextruder at $230^{\circ} \mathrm{C}$. The blending ratio of SAN to C5-DC12 was 80/20 wt \%. And as an antioxidant, $0.3 \mathrm{phr}$ of Irganox 1076 was added. After blending, the sample was ultramicrotomed and stained by an osmium tetraoxide $\left(\mathrm{OsO}_{4}\right)$ solution to make the rubber particle dark. TEM image shows the dark line on the surfaces of g-ABS particles as shown in Figure 5a. The black lines correspond to the silicate layers. To clarify whether the silicate is on the surface or in the particles, the silicate $(\mathrm{Si})$ analysis by EDAX was made for the C5-DC12/SAN blend as shown in Figure 5b. The peak at $1.7 \mathrm{keV}$ is characteristics for $\mathrm{Si}$ and $\mathrm{Si}$ peak appears on the 

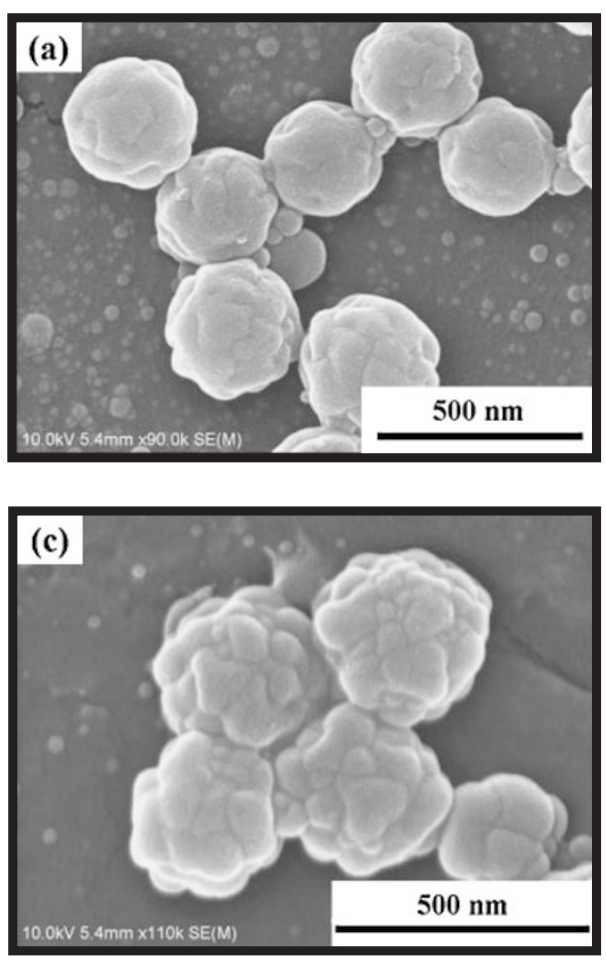

Figure 3. SEM images of the synthesized emulsion particles: (a) g-ABS (CO-NO), (b) C0-DC12, (c) C5-NO, and (d) C5-DC12.

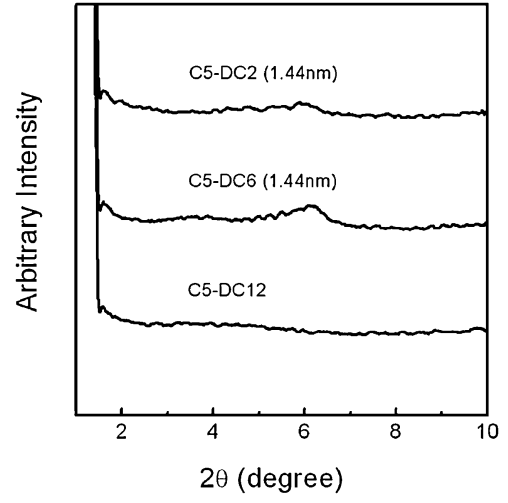

Figure 4. XRD patterns of $A B S / l a y e r e d$ silicate nanocomposites with $D C 2$, DC6, and DC12 at the constant loading of Cloisite-Na, 5 PHM.

surface of the particles, denoted by (a) in Figure 5b. The presence of $\mathrm{Si}$ peak indicates that most of silicate layers are attached on the surfaces of g-ABS particles owing to the hydrophobic character of reactive amphiphile.

\section{Thermal Properties}

Thermogravimetric analysis (TGA) of ABS/layered silicate nanocomposites is shown in Figure 6 and their residues and temperatures at $20 \%$ weight loss are summarized in Table II. The onset temperatures of thermal decomposition of nanothat of the neat ABS copolymer. The neat ABS copolymer showed $20 \%$ weight loss at $410{ }^{\circ} \mathrm{C}$, but $\mathrm{ABS} /$ layered silicate nanocomposites decomposed at $15-34{ }^{\circ} \mathrm{C}$ higher temperature. composites moved toward a higher temperature compared with
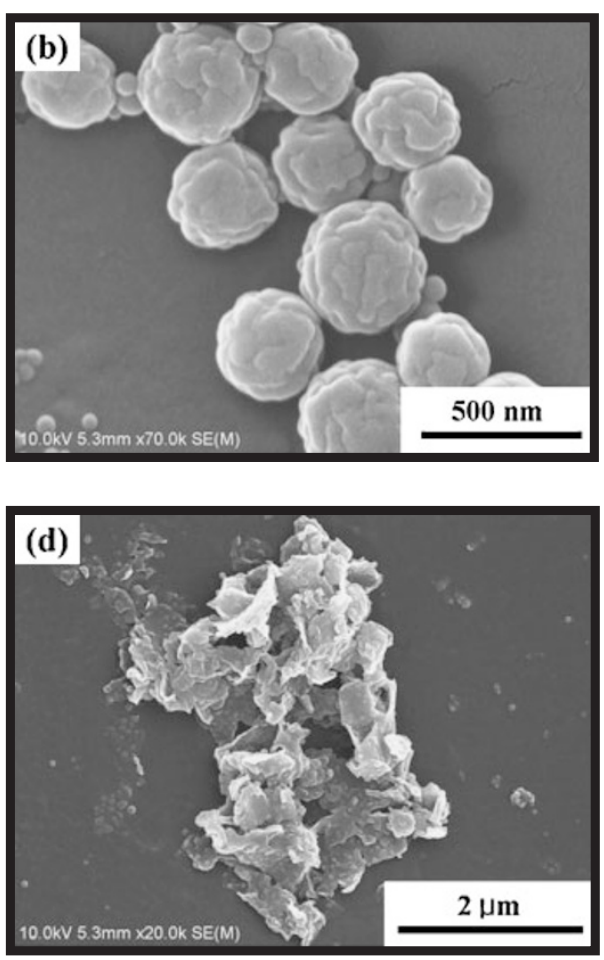
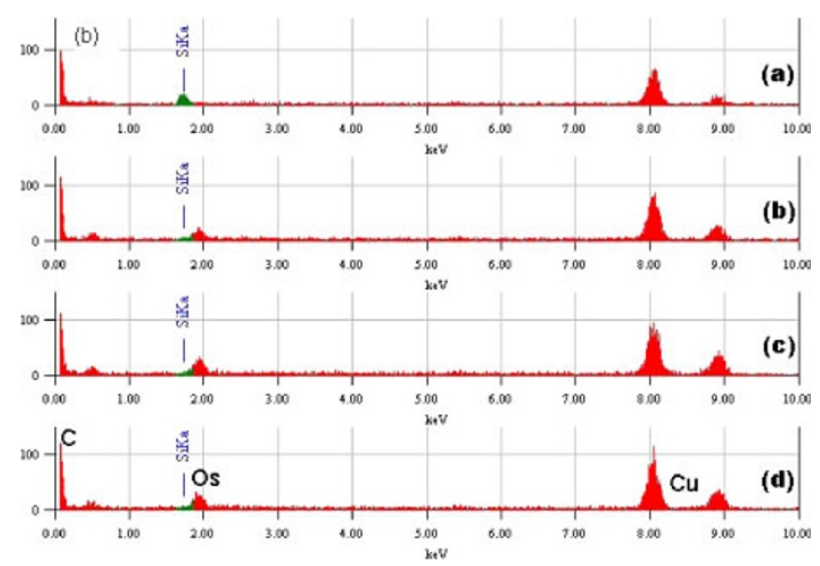

Figure 5. TEM images and EDAX curves of the C5-DC12/SAN blend.

And as shown in the Figure 6a, the onset of decomposition shifts toward a higher temperature as the amount of silicate increases in the samples with DC12. This may result from 

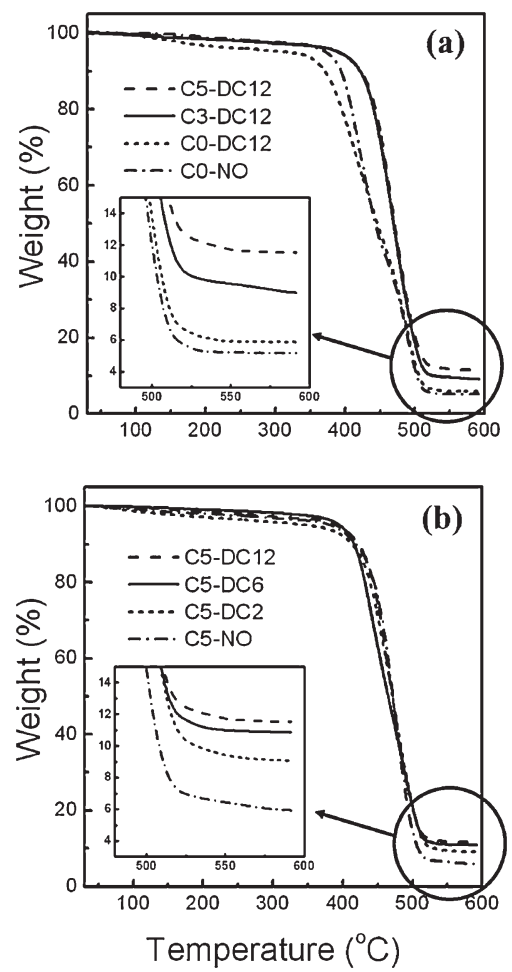

Figure 6. TGA curves of the synthesized ABS/layered silicate nanocomposite and neat $A B S$ copolymer under $\mathrm{N}_{2}$ atmosphere: (a) variation of silicate loading with $\mathrm{DC} 12$ and (b) change of reactive amphiphiles with constant silicate contents, 5 PHM.

Table II. Temperatures at $20 \%$ weight loss and residues of the synthesized materials

\begin{tabular}{cccc}
\hline Sample code & $\begin{array}{r}\mathrm{T} \text { at 20\% loss } \\
\left({ }^{\circ} \mathrm{C}\right)\end{array}$ & $\begin{array}{c}\text { Residue } \\
(\%)\end{array}$ & $\begin{array}{c}\text { Residue difference* } \\
(\%)\end{array}$ \\
\hline C0-NO & 410 & 5.20 & - \\
C0-DC12 & 395 & 5.88 & 0.68 \\
C5-NO & 425 & 5.93 & 0.73 \\
C5-DC2 & 439 & 9.07 & 3.87 \\
C5-DC6 & 432 & 10.85 & 5.65 \\
C3-DC12 & 441 & 9.04 & 3.84 \\
C5-DC-12 & 444 & 11.52 & 6.32
\end{tabular}

${ }^{*}$ Residue differences were obtained by subtraction the residue value of CO-NO from each residue.

the thermal barrier property of silicate platelets in the ABS copolymer matrix.

The amount of silicate contained in the ABS matrix was estimated from the residue differences in the thermograms and are listed in Table II. C5-NO has a very deficient residue because no reactive amphiphile was added to make silicates hydrophobic and a large portion of hydrophilic silicates floating in the water medium of latex was washed out during coagulation process. And it was found that the exfoliated nanocomposite prepared with DC12 produced larger residue difference than the intercalated ones with DC2 and DC6. This fact indicates that the exfoliated structure of silicate layers is more effective from the point of char forming. According to many studies, ${ }^{34-36}$ the lower flammability of PLSNs is not due to retention of a large fraction of fuel, but to the formation of carbonaceous char in the condensed phase. So the exfoliated nanocomposites give higher residue indicating the enhancement of char formation on the surface of ABS.

\section{CONCLUSIONS}

From the above results, ABS/layered silicate nanocomposites obtained by using reactive amphiphiles (DC2 or DC6) with short hydrophobic chains showed intercalated states of silicates. In the case of ABS/layered silicate nanocomposites made with longer reactive amphiphile (DC12), on the other hand, we confirmed that the silicates were exfoliated in ABS matrix and most of them were attached on the surface of the g-ABS particles. Based on the TGA analysis, the exfoliated nanocomposites gave higher residue indicating the enhancement of char formation. Finally it could be concluded that the reactive amphiphile made silicates adsorb on the polymer surfaces and enhanced the dispersibility of silicate and thermal stability in ABS/layered silicate nanocomposite.

Acknowledgment. The authors would like to express their sincere thanks to Samsung Cheil Industry for supporting this project. And, this work was also supported in part by Brain Korea 21 program.

Received: October 25, 2007

Accepted: December 10, 2007

Published: February 5, 2008

\section{REFERENCES}

1. P. B. Messersmith and E. P. Giannelis, J. Polym. Sci., Part A: Polym. Chem., 33, 1047 (1995).

2. A. Usuki, Y. Kojima, M. Kawasumi, A. Okada, Y. Fukushima, T. Kurauchi, and O. Kamigaito, J. Mater. Res., 8, 1179 (1993).

3. Y. Kojima, A. Usuki, M. Kawasumi, A. Okada, Y. Fukushima, T. Kurauchi, and O. Kamigaito, J. Mater. Res., 8, 1185 (1993).

4. K. Yano, A. Usuki, A. Okada, T. Kurauchi, and O. Kamigaito, J. Polym. Sci., Part A: Polym. Chem., 31, 2493 (1993).

5. R. A. Vaia, K. D. Jandt, E. J. Kramer, and E. P. Giannelis, Macromolecules, 28, 8080 (1995).

6. L. Biasci, M. Aglietto, G. Ruggeri, and F. Ciardelli, Polymer, 35 3296 (1994).

7. M. Alexandre and P. Dubois, Mater. Sci. Eng., R, 28, 1 (2000).

8. P. C. LeBaron, Z. Wang, and T. J. Pinnavaia, Appl. Clay Sci., 15, 11 (1999).

9. "Polymer-Clay Nanocomposites," T. J. Pinnavaia and G. W. Beall, Ed., Baffin Lane, John Wiley \& Sons Ltd, 2001.

10. P. B. Messersmith and E. P. Giannelis, J. Polym. Sci., Part A: Polym. Chem., 33, 1047 (1995).

11. S. D. Burnside, H. C. Wang, and E. P. Giannelis, Chem. Mater., 11, 1055 (1999).

12. Y. C. Kim, S. J. Lee, J. C. Kim, and H. Cho, Polym. J., 37, 206 (2005).

13. Y. C. Kim, Polym. J., 38, 250 (2006).

14. C. D. Muzny, B. D. Butler, H. J. Hanley, F. Tsvetkov, and D. G. Peiffer, Mater. Lett., 28, 379 (1996).

15. D. C. Lee and W. J. Lee, J. Appl. Polym. Sci., 61, 1117 (1996).

16. M. Laus, M. Camerani, M. Lelli, K. Sparnacci, F. Sandrolini, and O. J. Francescangeli, Mater. Sci., 33, 2883 (1998). 
17. S. S. Ray and M. J. Biswas, J. Appl. Polym. Sci., 73, 2971 (1999).

18. J. S. Bergman, H. Chen, E. P. Giannelis, M. G. Thomas, and G. W. Coates, Chem. Commun., 2179 (1999).

19. Z. Wang, T. Lan, and T. J. Pinnavaia, Chem. Mater., 8, 2200 (1996).

20. Z. Wang and T. J. Pinnavaia, Chem. Mater., 10, 1820 (1998).

21. C. Zilg, R. Mühaupt, and J. Finter, Macromol. Chem. Phys., 200, 661 (1999).

22. T. J. Pinnavaia, Science, 220, 365 (1983).

23. H. H. Murray, Appl. Clay. Sci., 17, 207 (2000).

24. R. A. Vaia, R. K. Teukosky, and E. P. Giannelis, Chem. Mater., 6, 1017 (1994).

25. R. Krishnamoorti, R. A. Vaia, and E. P. Giannelis, Chem. Mater, 8 , 1728 (1996).

26. R. Krishnamoorti and E. P. Giannelis, Macromolecules, 30, 4097 (1997).

27. R. Limary, S. Swinnea, and P. F. Green, Macromolecules, 33, 5227 (2000).
28. J. Ren, A. S. Silva, and R. Krishnamoorti, Macromolecules, 33, 3739 (2000).

29. D. M. Kulich, P. D. Kelly, and J. E. Pace, "Encyclopedia of polymer Science and Engineering," John Wiley and Sons, New York, New York, 1985.

30. "Handbook of Plastic Materials and Technology," I. I. Rubin, Ed., Wiley, New York, 1990.

31. L. W. Jang, M. K. Chul, and D. C. Lee, J. Polym. Sci., Part B Polym. Phys., 39, 719 (2001).

32. S. F. Wang, Y. Hu, L. Song, Z. Z. Wang, Z. Y. Chen, and W. C. Fan, Polym. Degrad. Stab., 77, 423 (2002).

33. Y. S. Choi, M. Xu, and I. J. Chung, Polymer, 46, 531 (2005).

34. J. Zhu and C. A. Wilkie, Polym. Int., 49, 1158 (2000).

35. J. W. Gilman, C. L. Jackson, and A. B. Morgan, Chem. Mater., 12, 1866 (2000).

36. R. A. Vaia, G. Price, P. N. Ruth, H. T. Nguyen, and J. Lichtenhan, Appl. Clay Sci., 15, 67 (1999). 